\title{
Elastoplastic model for unsaturated, quasi-saturated and fully saturated fine soils
}

\author{
Ba Tien Lai ${ }^{1}$, Antonin Fabbri ${ }^{1, a}$, Henry Wong ${ }^{1}$ Tuan Anh Bui ${ }^{1}$ and Denis Branque ${ }^{1}$ \\ ${ }^{1}$ Université de Lyon/ENTPE/LGCB-LTDS (UMR CNRS 5513) 3 rue Maurice Audin, 69120 Vaulx en Velin, France
}

\begin{abstract}
In unsaturated soils, the gaseous phase is commonly assumed to be continuous. This assumption is no more valid at high saturation ratio. In that case, air bubbles and pockets can be trapped in the porous network by the liquid phase and the gas phase becomes discontinuous. This trapped air reduces the apparent compressibility of the pore fluid and affect the mechanical behavior of the soil. Although it is trapped in the pores, its dissolution can take place. Dissolved air can migrate through the pore space, either by following the flow of the fluid or by diffusion. In this context, this paper present a hydro mechanical model that separately considers the kinematics and the mechanical behavior of each fluid species (eg liquid water, dissolved air, gaseous air) and the solid matrix. This new model was implemented in a $\mathrm{C}++$ code. Some numerical simulations are performed to demonstrate the ability of this model to reproduce a continuous transition of unsaturated to saturated states.
\end{abstract}

\section{Introduction}

An unsaturated soil is conceptually a tri-phasic medium, composed by a solid skeleton (S) and a porous network partially saturated by a liquid phase (L) while its remaining space is filled by a gas phase (G). At low to moderate water contents, both gaseous and liquid phases are continuous. The interaction between these two phases and the solid grain leads to a well-known suction effect which has the effect of increasing the shear strength and the rigidity of soils. In the recent years, numerous researches ([1], [2], [3], [4], [5], [6]) have focused on the modeling of unsaturated soil behavior. They have led to enhance classical elasto-plastic models (like CamClay) by bringing the influences of non-saturation. However few researches have studied the transition zone between the unsaturated and the fully saturated domains.

Experiments results on natural fine soils in temperate region or in compacted soils at the Optimum Proctor show that, , when the degree of saturation reaches values of $75-85 \%$, air bubbles and pockets are trapped within the continuous water phase. This domain is called "quasisaturated" by authors such as [8], [9],[10].

In the literature, some approaches to describe the coexistence of saturation domain such as [11], [12], [13] exist. However, the above models cannot account for the coupled hydromechanical behaviors and the continuous transition between different regimes of saturation.
A rigorous theoretical framework that is well-adapted to the formulation of coupled multi-physics problems in porous media would be necessary to simulate the existence of different regimes of saturation, as well as the evolution relative to space and time of the corresponding physical domains. To that end, an original model is proposed in this paper. It is part of an ongoing research project named Terredurable, which aims to provide a clearer understanding of coupled hydromechanical responses of civil engineering structures at close-tosaturation states.

\section{The new description of saturation domain}

In this approach, we account for three different domains of saturation: the unsaturated domain where both liquid and gas phases are continuous (US); the quasi-saturated domain where the gas phase in the form of bubbles is discontinuous (QS); and the fully saturated domain where air is only present in dissolved form (FS).

In order to arrive at a consistent description of a continuous transition across different saturation domains, the porous space occupied by the gaseous phase will conceptually be divided into two sets that can freely exchange within each other. The first, named "displaceable air" is the part that will be invaded by water during the wetting process before the loss of connectivity

\footnotetext{
${ }^{a}$ Corresponding author: antonin.fabbri@entpe.fr
} 


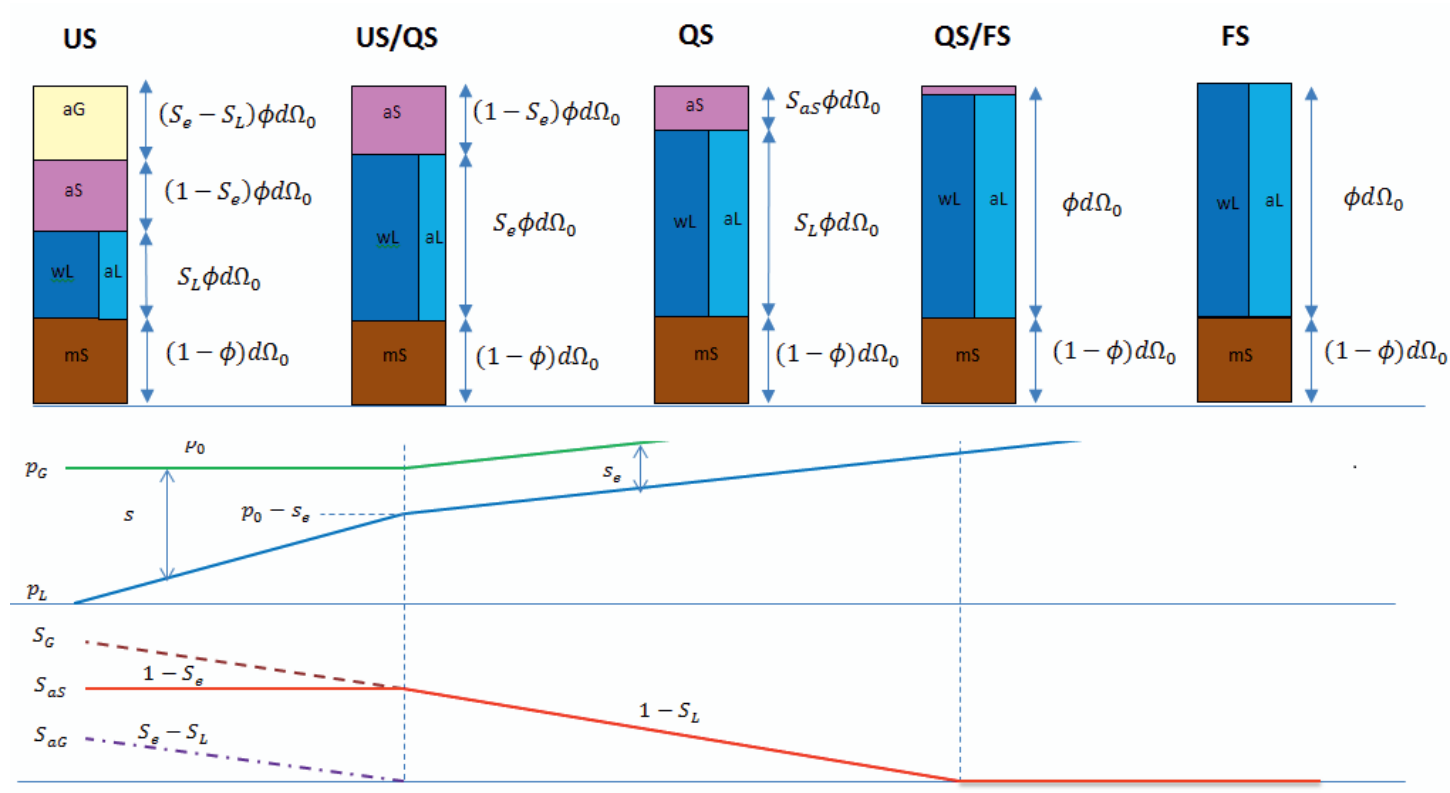

Figure 1. Transition between different domains of saturation during wetting.

of the gaseous phase; this volume is noted "aG". The remaining part, called "non-displaceable air", corresponds to locations where the air is trapped when the saturation attains the threshold $\mathrm{S}_{\mathrm{e}}$ The air within this volume is noted "aS". As mentioned previously, the capital "S" is used to emphasize the fact that the air within this volume has the same kinematics as the solid matrix. A simplified description of the saturation domains and the continuous transition assumed here is summarized in Figure 1.

In the US-domain, the gaseous phase is continuous and connected to outside; the difference between the gas pressure (equal to atmospheric pressure here) and the liquid pressure, defined as matric suction, is higher than the air-entry suction. The two sets of gaseous air, "aS" and "aG", are simultaneously present, physically connected to each other and share a common pressure equal to the atmospheric pressure:

$$
p_{0}-p_{L} \geq s_{e} ; p_{G}=p_{a G}=p_{0}
$$

This implicitly implies that the flow of gas towards the external boundary surface is instantaneous. Note that, by hypothesis, the degree of saturation corresponding to the air set "aS" remains constant in this phase: $S_{a S}=1-S_{e}$. Suction and degree of saturation are linked to each other via the celebrated equation of [14] for saturation ratios below $S_{e}: S_{L}=S_{e}\left(s / s_{e}\right)^{-\alpha}$.

In the QS-domain, suction is below the air-entry value and the gaseous phase is discontinuous; air is only present in the form of bubbles.

Being non-connected to the outside, pressure in the air bubbles can now exceed the atmospheric pressure. Note that the gas pressure is continuous at the transition $\mathrm{US} / \mathrm{QS}$, equal to $p_{0}$. In summary:

$$
p_{0}-p_{L}<s_{e} ; S_{a G}=0 ; S_{a S}>0 ; p_{G}=p_{L}+s_{e}>p_{0}
$$

The QS-domain is reached when all the trapped air is dissolved, the material becomes fully saturated. The mathematical conditions is $S_{a S}=0$.

\section{Mathematical formulations}

\subsection{Rheological constitutive equations}

In the model, the classic Camclay variables are used, namely the mean effective stress $p^{\prime}=\operatorname{tr}\left(\sigma^{\prime}\right) / 3$, and the equivalent deviatoric stress $q=\sqrt{(3 / 2) s: s}$ where $\boldsymbol{s}=\boldsymbol{\sigma}^{\prime}-\mathbf{1} \cdot p^{\prime} / 3$ is the deviatoric stress tensor. $\varepsilon_{p_{1}}=\operatorname{tr}(\varepsilon)$ is the volumetric strain while $\varepsilon_{q}=((2 / 3) \boldsymbol{e}: \boldsymbol{e})^{1 / 2}$ is the equivalent deviatoric strain, with $e=\varepsilon-1 \cdot \varepsilon_{p} / 3$.

The effective stress concept is used:

$$
\boldsymbol{\sigma}=\sigma^{\prime}-\mathbf{1} \cdot \pi
$$

where $\boldsymbol{\sigma}$ is the total stress tensor, $\mathbf{1}$ the second order identity tensor and $\pi$ the equivalent pore pressure. Several definitions for $\pi$ have been proposed, for example [15], [16]. The following form, proposed by Bishop, is used in this study:

$$
\pi=-p_{G}+S_{L}\left(p_{G}-p_{L}\right)
$$

We also assume that under isotropic virgin compression, the volumetric strain will vary linearly with the logarithm of the mean effective stress with a slope equal to:

$$
\lambda(s)=\left\{\begin{array}{cl}
\lambda_{0}[(1-r)] e^{-\beta s}+r & : \quad s>s_{e} \\
\lambda_{0} & : \quad s \leq s_{e}
\end{array}\right.
$$

where $\beta, r$ are model parameters 
We therefore adopt a simple isotropic logarithmic elastic behaviour like Camclay. The strain rates are entirely defined by the stress rates:

$$
\left(\begin{array}{c}
d \varepsilon_{p}^{e} \\
d \varepsilon_{q}^{e}
\end{array}\right)=\left(\begin{array}{c}
d p^{\prime} / K \\
d q /(3 G)
\end{array}\right) ; \quad K=\frac{1+e_{0}}{\kappa} p^{\prime} ; G=\frac{3(1-v)}{2(1+v)} K
$$

where where $K$ and $G$ are the state-dependent bulk and shear moduli, $v$ the Poisson ratio and $\kappa$ a material constant.

The loading surface adopted, is described by:

$$
f\left(p^{\prime}, q, p_{c}\right)=q^{2}-M^{2} p^{\prime}\left(p_{c}-p^{\prime}\right)
$$

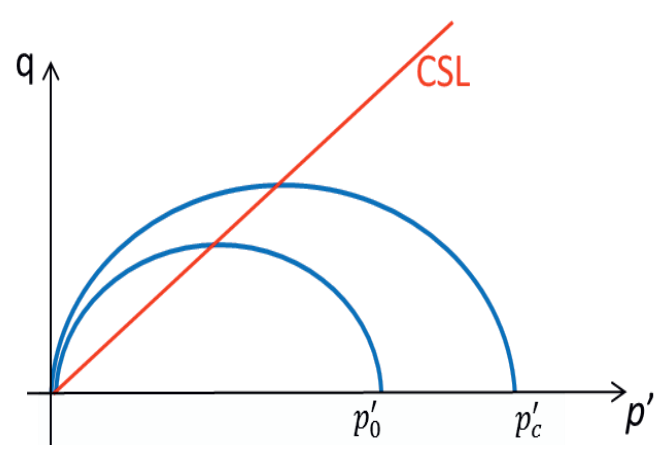

Figure 2. Loading surface in the plan (p',q).

The dependence of $p_{c}$ on suction can be described by:

$$
p_{c}= \begin{cases}p_{r e f}\left(\frac{p_{0}}{p_{r e f}}\right)^{\frac{\lambda_{0}-\kappa}{\lambda(s)-\kappa}}: & s>s_{e} \\ p_{0} & : s \leq s_{e}\end{cases}
$$

$p_{0}$ is the hardening variable in the case of full saturation, similar to Camclay, verifying:

$$
d p_{0}=\frac{1+e_{0}}{\lambda_{0}-\kappa} p_{0} d \varepsilon_{p}^{p}
$$

It is postulated that plastic strain rates write:

$$
\left(\begin{array}{l}
d \varepsilon_{p}^{p} \\
d \varepsilon_{q}^{p}
\end{array}\right)=d \lambda\left(\begin{array}{l}
\frac{\partial f}{\partial p^{\prime}} \\
\frac{\partial f}{\partial q}
\end{array}\right)
$$

where $d \lambda=\frac{1}{H}\left(\frac{\partial f}{\partial p^{\prime}} d p^{\prime}+\frac{\partial f}{\partial q} d q+\frac{\partial f}{\partial s} d s\right)$

Where $d \lambda$ plastic multiplier and $\mathrm{H}$ plastic modulus.

$$
H=\frac{v p^{\prime 3}\left(M^{4}-\eta^{4}\right)}{\lambda(s)-\kappa}
$$

fraction of dissolved air being much lower than that of liquid water, the mass balance of liquid water writes:

$$
\frac{\partial S_{L}}{\partial t}+\frac{\phi S_{L}}{K_{L}} \frac{\partial p_{L}}{\partial t}+S_{L} \frac{\partial \phi}{\partial t}=\nabla \cdot\left(D_{L} \nabla p_{L}-D_{L} \rho_{L}^{0} \boldsymbol{g}\right)
$$

where $\rho_{L}^{0}$ the liquid density, $D_{L}$ the permeability.

The mass balance of dissolved air is due to three main contributions: (a) the advective transport of dissolved air within the liquid phase; (b) the diffusion of the dissolved air within the liquid phase; and (c) exchange with gaseous air via the dissolution/bubbling process. After some detailed developments, we get:

$$
\begin{aligned}
& \phi S_{L} \frac{\partial m}{\partial t}+m\left(\frac{\phi S_{L}}{K_{L}} \frac{\partial p_{L}}{\partial t}+\phi \frac{\partial S_{L}}{\partial t}+S_{L} \frac{\partial \phi}{\partial t}\right) \\
& =\nabla \cdot\left[m D_{L}\left(\nabla p_{L}-\rho_{L} \boldsymbol{g}\right)+D_{e} \nabla m\right]+\frac{\hat{m}_{G \rightarrow a L}}{\rho_{L}}
\end{aligned}
$$

where $D_{e}$ is the effective diffusion coefficient of dissolved air, $\hat{m}_{G \rightarrow a L}$ the mass rate of dissolution/bubbling of air within the liquid phase. $m=m_{a L} / m_{w L}$ the mass of dissolved air per unit of liquid water mass. When the air in the gaseous state still exists within the REV, $\mathrm{S}_{\mathrm{L}}<1$ we have $m=m^{e q}$ where $m^{e q}$ is the mass of dissolved air per unit of liquid water mass at equilibrium with the entrapped air pockets. $m^{e q}$ is calculated by the Henry's law:

$$
p_{a S}=K_{H} \frac{M_{L}}{M_{G}} m^{e q}
$$

To derive the equation of mass balance for gaseous air, we assume that the in-pore air phase follows the law of perfect gas:

$m_{a G}=\frac{\phi S_{a G} p_{a G} M_{G}}{R T} ; m_{a S}=\frac{\phi S_{a S} p_{a S} M_{G}}{R T}$

With $R$ the universal gas constant and $T$ the absolute temperature. Neglecting evaporation and condensation phenomena, the general form of the mass conservation of gaseous air is:

$$
\frac{\partial\left(m_{a G}+m_{a S}\right)}{\partial t}=\nabla \cdot \boldsymbol{\omega}_{G}-\hat{m}_{G \rightarrow a L}
$$

where $\nabla \cdot \omega_{G}$ stands for the mass in-flux due to the Darcean flow of gas through the porous network. To advance further, we need to inject the expressions of various quantities into equations (12), (13) and (16) and simplify. After a lengthy manipulation, we obtain the following three equations:

\subsection{Masse balances equations}

There are three independent mass balance equations, on liquid water, gaseous air and dissolved air. The mass 
$A_{1} \frac{\partial p_{L}}{\partial t}+A_{2} \frac{\partial e}{\partial t}-n \frac{\partial S_{a S}}{\partial t}=\nabla \cdot\left(D_{L} \nabla p_{L}\right)$

$\phi S_{L} \frac{\partial m}{\partial t}+A_{3} \frac{\partial p_{L}}{\partial t}+A_{4} \frac{\partial e}{\partial t}+\phi\left(\beta_{1} \frac{\rho_{G}}{\rho_{L}}-m\right) \frac{\partial S_{a S}}{\partial t}$

$=\nabla \cdot\left[m D_{L} \nabla p_{L}+D_{e} \nabla m\right]$

$\phi\left(m_{e q}-\beta_{1} \frac{\rho_{G}}{\rho_{L}}\right) \frac{\partial S_{a S}}{\partial t}$

$=\beta_{2}\left[A_{5} \frac{\partial p_{L}}{\partial t}+A_{6} \frac{\partial e}{\partial t}-\nabla \cdot\left[m D_{L} \nabla p_{L}+D_{e} \nabla m\right]\right]$

With

$A_{1}=\phi\left[\frac{S_{L}}{K_{L}}-\frac{d S_{a G}}{d p_{L}}\right] ; A_{2}=\phi\left[-\frac{d S_{a G}}{d e}+\frac{S_{L}}{e}\right] ;$

$A_{3}=\phi\left(m \frac{S_{L}}{K_{L}}-m \frac{d S_{a G}}{d p_{L}}+\beta_{1} \frac{\rho_{G}}{\rho_{L}} \frac{S_{a S}}{p_{G}}\right)$;

$A_{4}=\phi\left[m \frac{S_{L}}{e}-m \frac{d S_{a G}}{d e}+\beta_{1} \frac{\rho_{G}}{\rho_{L}} S_{a S}\left(\frac{1}{e}\right)\right]$;

$A_{5}=\phi\left[\left(m_{e q} S_{L}+\frac{\rho_{G}}{\rho_{L}} S_{a S}\right) \frac{\beta_{1}}{p_{G}}+m_{e q} \frac{S_{L}}{K_{L}}-m_{e q} \frac{d S_{a G}}{d p_{L}}\right]$;

$A_{6}=\phi\left[\left(m_{e q} S_{L}+\frac{\rho_{G}}{\rho_{L}} S_{a S}\right)\left(\frac{1}{e}\right)-m_{e q} \frac{d S_{a G}}{d e}\right]$

\section{Numerical results}

To illustrate the consistency and the applicability of this model, we present in this section a few numerical examples. We consider a 1D configuration of a homogeneous soil sample of $5 \mathrm{~cm}$ thick in an oedometric cell. Initially, the sample is in thermodynamic and mechanical equilibrium and is in an unsaturated state (US). The sample is then subjected to a monotonic increase of liquid pressure at the "bottom" boundary surface $(x=0)$, from $-250[\mathrm{kPa}]$ to $650[\mathrm{kPa}]$ in $150[\mathrm{~s}]$. At the top boundary $(x=5 \mathrm{~cm})$, there is no flux. The total stress is kept constant equal to its initial value $\sigma_{0}=1 \mathrm{MPa}$. Radial displacement and radial flow are null. This simulation corresponds to a progressive wetting of a sample. The parameters used for the simulation are summarized in Appendix. Finally, we assume that the mass concentration of dissolved air in the incoming liquid water is, at any time, equal to the concentration of dissolved air within the pores of the sample which are directly in contact with the external surfaces. In summary, we have:

$$
\begin{aligned}
& \sigma_{x}(x=0, t)=\sigma_{0} ; u_{x}(x=L, t)=0 ; \\
& \nabla m \cdot \boldsymbol{e}_{x}(x=0, t)=\nabla m \cdot \boldsymbol{e}_{x}(x=L, t)=0 \\
& p_{L}(x=0, t)=p_{L 0}+\delta p_{L} \frac{t}{t_{\text {final }}}
\end{aligned}
$$

For $\mathrm{t}=35[\mathrm{~s}]$ the whole sample is unsaturated (US). The liquid saturation profile is directly linked to the liquid pressure (which is synonymous with suction, given the constant air pressure) through the retention curve. In this domain, the entrapped air saturation ratio is constant. (c.f. Figures $3 \& 4$ ).

For $\mathrm{t}=85[\mathrm{~s}]$ a transition between the unsaturated and the quasi-saturated domain is observed at, $\mathrm{x}=0.0105[\mathrm{~m}]$. This transition can easily be observed in Figure 3, where $\mathrm{S}_{\mathrm{aS}}$ remains equal to $1-\mathrm{S}_{\mathrm{e}}$ in the unsaturated domain, and progressively decreases from the US-QS.

Finally, at $\mathrm{t}=150[\mathrm{~s}]$ all three domains of saturation coexists: the unsaturated state in $0 \leq x \leq 0.002$ the quasisaturated state in $0.002[\mathrm{~m}]<x<0.0255[\mathrm{~m}]$ and the fully saturated state for $x \geq 0.0255$ [m] .

The variation of void ratio with liquid pressure and effective stress at $\mathrm{x}=0[\mathrm{~m}]$ are presented in the Figure 5 . Three different behaviours, delimited by the points A, B $\mathrm{C}$ and $\mathrm{D}$ in Figure 5, can be observed. From A to B, the material at $\mathrm{x}=0$ is in the unsaturated domain (the value of liquid pressure is less than $\mathrm{p}_{0}-\mathrm{S}_{\mathrm{e}}$ ), the behaviour of soils is elastic; from $\mathrm{B}$ to $\mathrm{C}$, it is in the quasi-saturated domain, the behaviour of soils is elastic; From $\mathrm{C}$ to $\mathrm{D}$, a variation of the slope of the relation $\sigma$ '-e is observed, the behaviour of soil becomes elastoplastic.

Furthermore, in the unsaturated domain, the void ratio variations with the increase of the liquid pressure remain limited. On the opposite, in the quasi-saturated state, an increase in a fluid pressure causes a sharp variation of the void ratio. This numerical result is consistent with experimental results of quasi-saturated soils of [17].

\section{Conclusions}

This paper presents a detailed study of the hydromechanical behaviours of compacted fine soils close to saturation. These soils are characterized by the presence of entrapped air. An original model capable of simulating the various couplings between physical and hydromechanical phenomena intervening in such type of soils is presented in this paper. The model developed is able to simulate a consistent description of the simultaneous presence of different domains of saturation, as well as their evolution relative to space and time.The numerical example allows checking the consistency of the model.

\section{Acknowledgement}

This work has been supported by the French National Research Agency through the projet TerreDurable (ANR11-VILD-0004). 

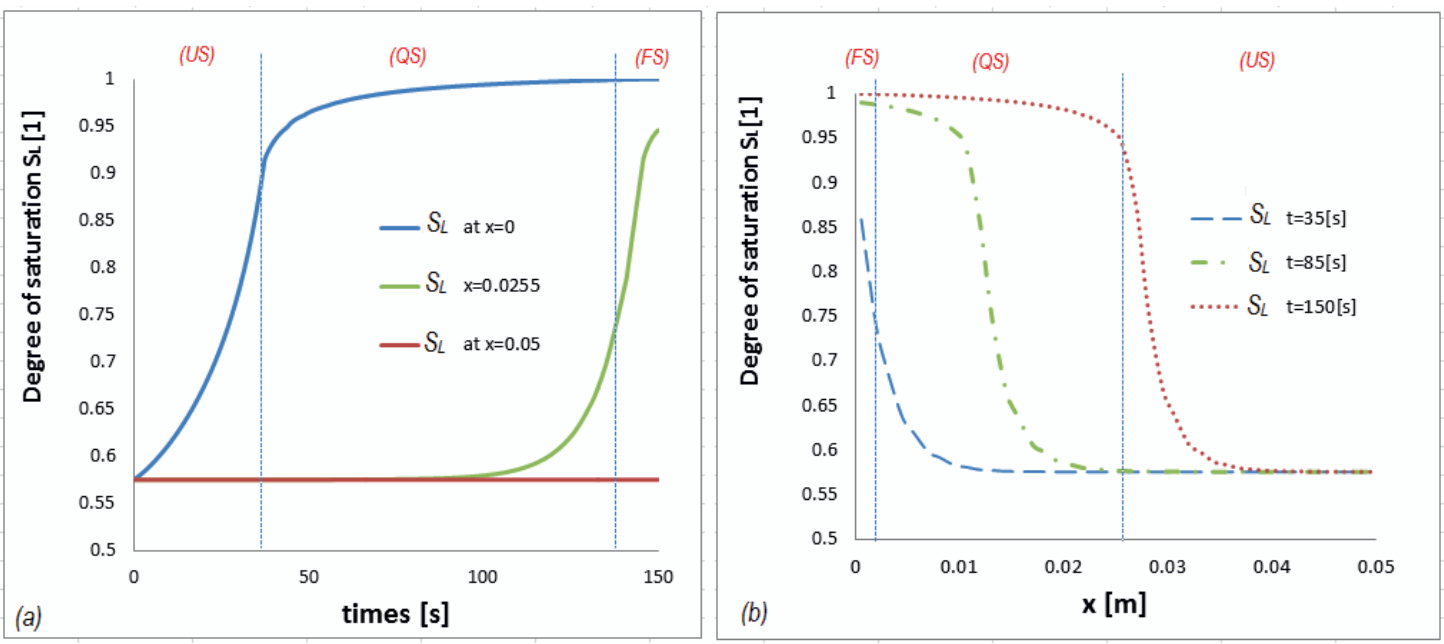

Figure 3. Variation of degree of saturation with (a) times (b) space
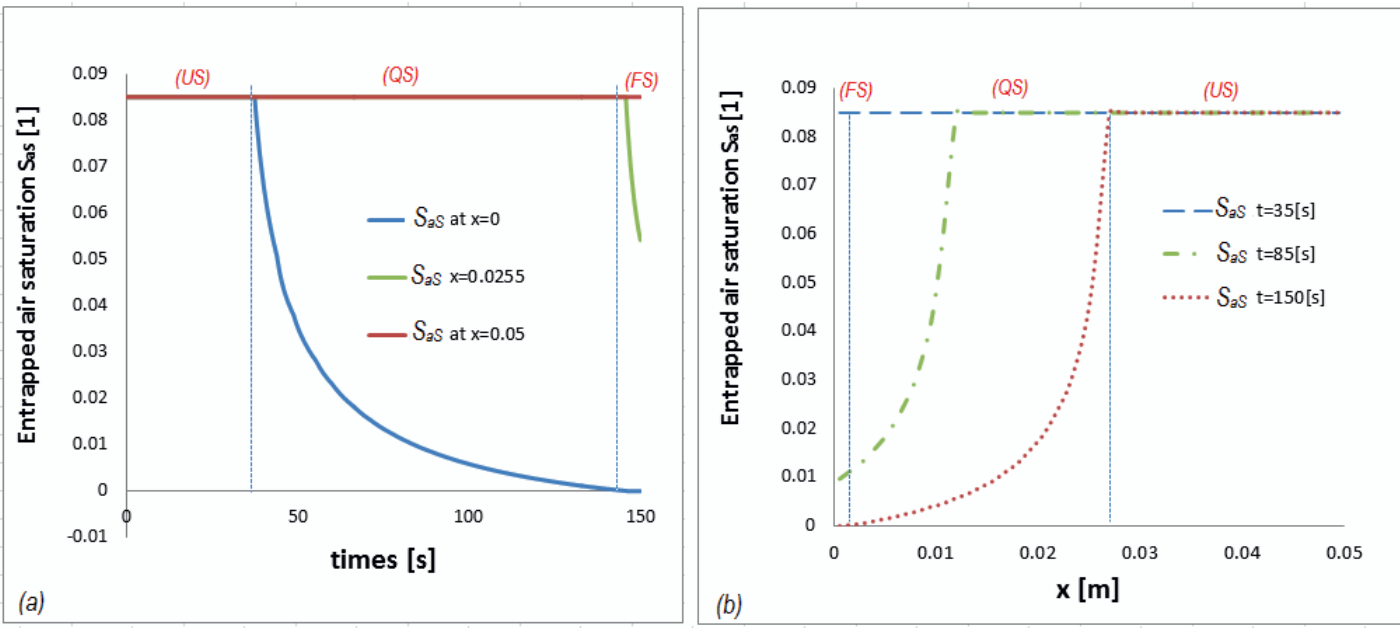

Figure 4. Variation of saturation of entrapped air with (a) times (b) space
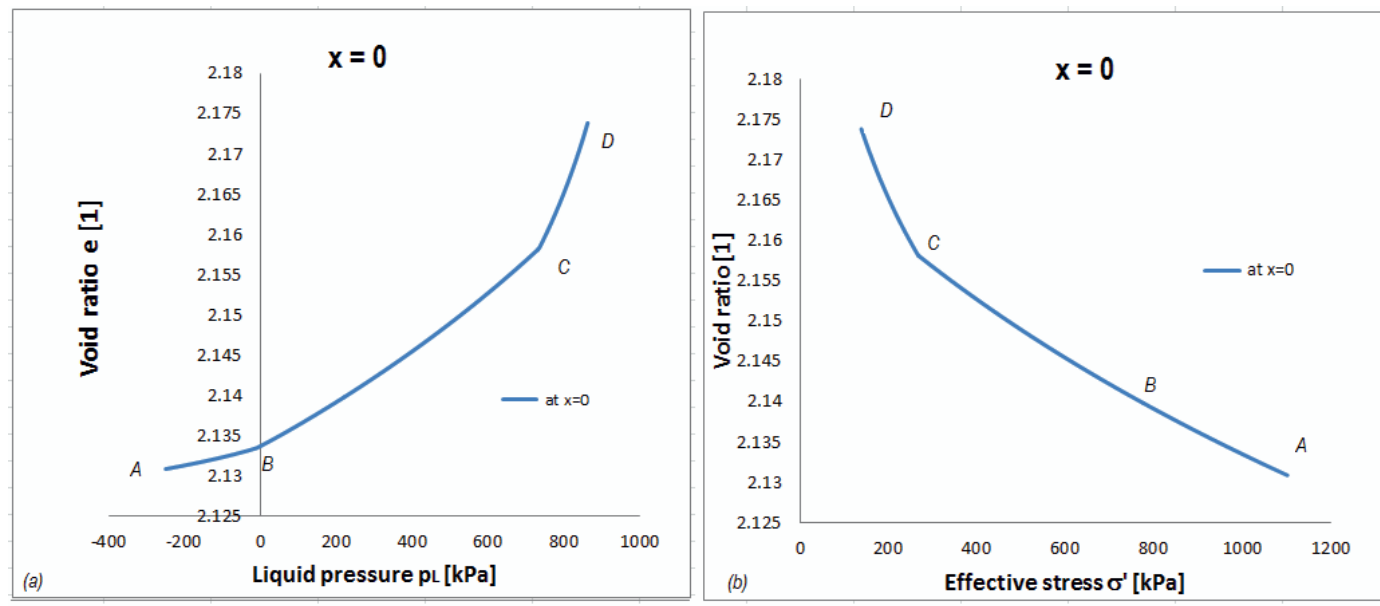

Figure 5. Variation of void ratio at $\mathrm{x}=0$ with (a) liquid pressure (b) effective stress 


\section{Appendix 1}

\begin{tabular}{|c|c|c|}
\hline Parameters & Description & $\begin{array}{l}\text { Value } \\
\text { [unit] }\end{array}$ \\
\hline$K_{H}$ & Henry' constant for air & $7326[\mathrm{MPa}]$ \\
\hline $\mathrm{R}$ & ideal gas constant & $\begin{array}{c}8.314 \\
{[\mathrm{~J} /(\mathrm{mol} . \mathrm{K})]}\end{array}$ \\
\hline$M_{L}$ & Molar mass of water & $18[\mathrm{~g} / \mathrm{mol}]$ \\
\hline$M_{G}$ & Molar mass of air & $29[\mathrm{~g} / \mathrm{mol}$ \\
\hline$p_{0}$ & Atmospheric air pressure & $100[\mathrm{kPa}]$ \\
\hline$T$ & Absolute temperature & $293[\mathrm{~K}]$ \\
\hline$K_{L}$ & Bulk modulus of water & $1970[\mathrm{MPa}]$ \\
\hline$\eta$ & $\begin{array}{c}\text { Dynamic viscosity of } \\
\text { water }\end{array}$ & $\begin{array}{c}10-3 \\
{[\mathrm{~kg} /(\mathrm{m} . \mathrm{s})]}\end{array}$ \\
\hline$D_{L}$ & Permeability & $8.10-9[\mathrm{~m} / \mathrm{s}]$ \\
\hline$e_{0}$ & Initial void ratio & $1.97[1]$ \\
\hline$S_{e}$ & $\begin{array}{c}\text { Degree of saturation at } \\
\text { air-entry }\end{array}$ & $0.915[1]$ \\
\hline$S_{e 0}$ & Initial air-entry suction & $110[\mathrm{kPa}]$ \\
\hline$\alpha$ & $\begin{array}{c}\text { Coefficient of retention } \\
\text { curve }\end{array}$ & $0.4[1]$ \\
\hline$\kappa$ & $\begin{array}{c}\text { Elastic stifness } \\
\text { parameters }\end{array}$ & $0.05[1]$ \\
\hline$\lambda_{0}$ & $\begin{array}{c}\text { Stiffness parameter in } \\
\text { virgin state of saturated } \\
\text { soils }\end{array}$ & $0.6[1]$ \\
\hline$\beta$ & Model parameter & $12.5\left[\mathrm{kPa}^{-1}\right]$ \\
\hline$r$ & Model parameter & $0.75[1]$ \\
\hline$p_{L 0}$ & Initial liquid pressure & $-250[\mathrm{kPa}]$ \\
\hline$\sigma_{0}$ & Initial total stresse & $1000[\mathrm{kPa}]$ \\
\hline$S_{L 0}$ & $\begin{array}{l}\text { Initial degree of } \\
\text { saturation }\end{array}$ & $0.566[1]$ \\
\hline$m_{0}$ & $\begin{array}{c}\text { Initial mass fraction of } \\
\text { dissolved air }\end{array}$ & $22.10^{-6}[1]$ \\
\hline
\end{tabular}

\section{References}

[1] E. E. Alonso, A. Gens, A. Josa, Géotechnique 40, 405-430 (1990).

[2] A. Modaressi, N. Abou-Berk, Proceedings of Numerical Methods in Geotechnical Engineering. (1994)

[3] H.Q. Pham, D.G. Fredlund, S.L. Barbour, Géotechnique, 53(2), 293-298 (2003)

[4] H.Q. Vu, D.G. Fredlund, Canadian Geotechnical Journal, 41(4), 713-737 (2004)

[5] E.E. Alonso, Revue Française de Géotechnique, 111, 3343 (2005)

[6] J-M. Pereira (2005). LGM, DGCB, ENTPE (2005)
[8] D. A. Stonestrom and J. Rubin J, Water resources research, 25(9), 1947-1958 (1989)

[9] B. Faybishenko, Water Resources Research, 31(10), 2421-2435 (1995)

[10] B.T. Lai, A. Fabbri, H. Wong, D. Branque, Computers and Geotechnics 69, 627-640 (2015)

[11]A. Denoth, Journal of Glaciology 28(99) 357-364 (1980)

[12]L. Boutonnier, Thèse à l'Institut National Polytechnique de Grenoble (2007)

[13]S.K. Vanapalli, D.G. Fredlund, \& D.E.Pufahl, Geotechnique, 49(2), 143-159 (1999)

[14]R. Brooks,A. Corey, Hydraulic properties of Porous Media. Colorado State University, (1964)

[15]O. Coussy, Poromechanics. John Wiley \& Sons.(2004),

[16] A. W. Bishop, Teknisk Ukeblad, 106(39), 859-863 (1963)

[17]J. M. Fleureau, rapport du LMSS.(2003). 